(n)

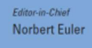
Journal of Nonlinear Mathematical Physics

\title{
New C-integrable and S-integrable systems of nonlinear partial differential equations
}

Francesco Calogero

To cite this article: Francesco Calogero (2017) New C-integrable and S-integrable systems of nonlinear partial differential equations, Journal of Nonlinear Mathematical Physics 24:1, 142-148, DOI: https://doi.org/10.1080/14029251.2017.1287387

To link to this article: https://doi.org/10.1080/14029251.2017.1287387

Published online: 04 January 2021 


\title{
New C-integrable and S-integrable systems of nonlinear partial differential equations
}

\author{
Francesco Calogero \\ Physics Department, University of Rome "La Sapienza", Italy \\ Istituto Nazionale di Fisica Nucleare, Sezione di Roma, Italy \\ francesco.calogero@romal.infn.it,francesco.calogero@uniroma1.it
}

Received 10 November 2016

Accepted 27 November 2016

\begin{abstract}
A technique to identify new C-integrable and S-integrable systems of nonlinear partial differential equations is reported, with two representative examples displayed and tersely discussed.

Keywords: systems of integrable partial differential equations, C-integrable PDEs, S-integrable PDEs
\end{abstract}

\section{Introduction}

The main tool used in this paper are the nonlinear reversible relations-by definition, algebraicamong the $N$ coefficients of a monic polynomial of degree $N$ in the (complex) variable $z$ and its $N$ zeros. The approach based on these relations allowed over time to identify many dynamical systems solvable by algebraic operations, including many-body problems characterized by Newtonian equations of motion ("accelerations equal forces") [1], and also several solvable/integrable systems of nonlinear Partial Differential Equations (PDEs) [2]. These developments were until recently mainly restricted to the consideration of nonlinear evolutions satisfied by the zeros of a time-dependent polynomial the coefficients of which evolve according to linear systems of Ordinary Differential Equations (ODEs) [1] or of PDEs [2]. Recently a convenient way to relate the time-evolution of the zeros of a time-dependent polynomial to the time-evolution of its coefficients has been noted [3], and this development has allowed the identification and investigation of several new solvable dynamical systems and many-body problems characterized by the time-evolution of the zeros of polynomials the coefficients of which evolve in a nonlinear but solvable/integrable manner [3,4]. In the present paper we show how this development can be as well employed to identify new systems of solvable/integrable nonlinear PDEs. Since our main goal in this paper is to introduce this approach we limit its application herein to the exhibition of just two new systems of integrable PDEs in 1+1 dimensions, the first of which is associated to the evolution of the $N$ zeros of a polynomial the coefficients of which evolve according to the Burgers PDE-perhaps the most elementary C-integrable nonlinear PDE in $1+1$ dimensions, being solvable by a Change of dependent variables-and the second of which is associated with the KdV PDE-perhaps the most famous of the nonlinear $S$ integrable PDEs in 1+1 dimensions, since the discovery half a century ago of its integrability via the Spectral (or Scattering) Transform opened the way to a major development in pure and applied mathematics. [5] 
Notation 1.1. Hereafter we always refer to monic polynomials of arbitrary order $N(N \geq 2)$,

$$
P_{N}(z ; \vec{\varphi}(x, t), \underline{\psi}(x, t))=z^{N}+\sum_{m=1}^{N}\left[\varphi_{m}(x, t) z^{N-m}\right]=\prod_{n=1}^{N}\left[z-\psi_{n}(x, t)\right]
$$

the complex variable $z$ is the argument of the polynomial, indices such as $n, m$ run throughout from 1 to $N$, the $N$-vector $\vec{\varphi}(x, t)$ has the $N$ coefficients $\varphi_{m}$ of the polynomial (1.1) as its $N$ components, $\underline{\psi}(x, t)$ denotes the unordered set of the $N$ zeros $\psi_{n}(x, t)$ of the polynomial (1.1), and we generally assume all these dependent variables to be complex (this of course does not exclude that they might be real, see indeed the examples below). We instead assume the independent variables $x$ ("space") and $t$ ("time ") to be real numbers; and we indicate partial differentiations with respects to these variables by appending them as subscripts preceded by commas, so for instance $\varphi_{m, t}(x, t) \equiv \partial \varphi_{m}(x, t) / \partial t, \psi_{n, x x}(x, t) \equiv \partial^{2} \psi_{n}(x, t) / \partial x^{2}$. We generally focus on generic polynomials the coefficients and zeros of which are generic complex numbers, and which in particular feature zeros all different among themselves, $\psi_{n}(x, t) \neq \psi_{m}(x, t)$ if $n \neq m$. Hereafter we often omit the explicit indication of the dependent variables $x$ and $t$ when this can be done without causing confusion. Note that the notation $P_{N}(z ; \vec{\varphi}, \underline{\psi})$ is somewhat redundant, since this monic polynomial of degree $N$ in $z$ can be identified by assigning either its $N$ coefficients $\varphi_{m}$ or its $N$ zeros $\psi_{n}$; indeed the $N$ coefficients $\varphi_{m}$ can be expressed in terms of the $N$ zeros $\psi_{n}$ via the standard formula

$$
\varphi_{m}=(-1)^{m} \sum_{1 \leq n_{1}<n_{2}<\ldots<n_{m} \leq N}\left(\psi_{n_{1}} \psi_{n_{2}} \cdots \psi_{n_{m}}\right)
$$

so that

$$
\begin{gathered}
\varphi_{1}=-\left(\psi_{1}+\psi_{2}+\ldots+\psi_{N}\right) \\
\varphi_{2}=\left(\psi_{1} \psi_{2}+\psi_{1} \psi_{3}+\ldots+\psi_{1} \psi_{N}\right) \\
+\left(\psi_{2} \psi_{3}+\psi_{2} \psi_{4}+\ldots+\psi_{2} \psi_{N}\right)+\ldots \\
+\left(\psi_{N-2} \psi_{N-1}+\psi_{N-2} \psi_{N}\right)+\psi_{N-1} \psi_{N},
\end{gathered}
$$

and so on. On the other hand, while the assignment of the $N$ coefficients $\varphi_{m}$ determines uniquely, up to permutations, the $N$ zeros $\psi_{n}$, of course explicit formulas in terms of elementary functions (including radicals) expressing the zeros of a polynomial of degree $N$ in terms of its coefficients are generally only available for $N \leq 4$. Finally let us note that hereafter we adopt the standard convention according to which $a$ void sum vanishes, and a void product equals unity.

In the following Section 2 we report and discuss our main findings, which are then proven in the following Section 3. A terse Section 4 outlines possible future developments. 


\section{Results}

Proposition 2.1. The following system of $N$ coupled nonlinear PDEs in $1+1$ variables is $C$ integrable:

$$
\begin{aligned}
\psi_{n, t}+\psi_{n, x x}= & \sum_{\ell=1, \ell \neq n}^{N}\left(\frac{2 \psi_{n, x} \psi_{\ell, x}}{\psi_{n}-\psi_{\ell}}\right) \\
& -\left[\prod_{\ell=1, \ell \neq n}^{N}\left(\psi_{n}-\psi_{\ell}\right)\right]^{-1} \sum_{m=1}^{N}\left[a_{m} \varphi_{m, x} \varphi_{m}\left(\psi_{n}\right)^{N-m}\right],
\end{aligned}
$$

where the parameters $a_{m}$ are $N$ arbitrary (complex) numbers, the $N$ (complex) functions $\psi_{n} \equiv$ $\psi_{n}(x, t)$ are the dependent variables, and the $N$ (complex) functions $\varphi_{m} \equiv \varphi_{m}(x, t)$ are expressed in terms of the dependent variables $\psi_{n} \equiv \psi_{n}(x, t)$ by the formulas (1.2), implying of course

$$
\begin{gathered}
\varphi_{1, x}=-\sum_{n=1}^{N}\left(\psi_{n, x}\right), \\
\varphi_{m, x}=(-1)^{m} \sum_{s=1}^{m}\left[\psi_{n_{s}, x} \cdot \sum_{1 \leq n_{1}<n_{2}<\cdots n_{s-1}<n_{s+1}<n_{m} \leq N}\left(\psi_{n_{1}} \psi_{n_{2}} \cdots \psi_{n_{s-1}} \psi_{n_{s+1}} \cdots \psi_{n_{m}}\right)\right] \\
m=2, \ldots, N .
\end{gathered}
$$

This means that the initial-value problem - to compute the $N$ functions $\psi_{n}(x, t)$ for all time $t>0$ from given initial data $\psi_{n}(x, 0)$ — can be solved by algebraic operations (including changes of variables from the coefficients to the zeros of a polynomial of degree $N$ such as (1.1)) and quadratures. The procedure to do so is detailed in the following Section 3, and this implies the validity of the solutions reported below.

For $N=2$ this system, (2.1), of 2 coupled nonlinear PDEs reads as follows:

$$
\begin{aligned}
\psi_{n, t}+\psi_{n, x x}= & \left(\psi_{n}-\psi_{n+1}\right)^{-1}\left\{2 \psi_{n, x} \psi_{n+1, x}+\left[a_{1}\left(\psi_{n, x}+\psi_{n+1, x}\right)\left(\psi_{n}+\psi_{n+1}\right) \psi_{n}\right]\right. \\
& \left.-a_{2}\left(\psi_{n, x} \psi_{n+1}+\psi_{n} \psi_{n+1, x}\right) \psi_{n} \psi_{n+1}\right\}, \quad n=1,2 \bmod (2) .
\end{aligned}
$$

An example of specific solution of this system of 2 coupled nonlinear PDEs, (2.3), reads as follows:

$$
\begin{gathered}
\psi_{n}(x, t)=-\frac{1+(-1)^{n}\left\{1-4\left[f_{1}(x, t)\right]^{2} / f_{2}(x, t)\right\}^{1 / 2}}{2 f_{1}(x, t)}, n=1,2, \\
f_{n}(x, t)=-\frac{a_{n}}{2 \gamma_{n}}+\beta_{n} \exp \left[-\gamma_{n}\left(x-\gamma_{n} t\right)\right], n=1,2,
\end{gathered}
$$

where the 2 parameters $a_{n}$ are those appearing in the PDEs (2.3) and the 4 (nonvanishing) parameters $\beta_{n}$ and $\gamma_{n}$ can be arbitrarily assigned. Note that if the 6 parameters $a_{n}, \beta_{n}, \gamma_{n}$ are all real numbers, then the 3 inequalities $a_{1} \beta_{1} \gamma_{1}<0, a_{2} \gamma_{2}>0, \beta_{2}<0$ are sufficient to guarantee that for all real values of the independent variables $x, t$ these solutions (2.4) are real and nonsingular. Also note that if $\gamma_{1}=\gamma_{2}=\gamma$ this solution has the "single soliton" feature to depend on the space and time coordinates only via their combination $x-\gamma t$. 
Proposition 2.2. The following system of $N$ coupled nonlinear PDEs in $1+1$ variables is $S$ integrable:

$$
\begin{aligned}
\psi_{n, t}+\psi_{n, x x x}= & 3 \sum_{\ell=1, \ell \neq n}^{N}\left(\frac{\psi_{n, x x} \psi_{\ell, x}+\psi_{n, x} \psi_{\ell, x x}}{\psi_{n}-\psi_{\ell}}\right)-3 \sum_{\ell_{1}, \ell_{2}=1 ; \ell_{1} \neq \ell_{2}, \ell_{1}, \ell_{2} \neq n}^{N} \frac{\psi_{n, x} \psi_{\ell_{1}, x} \psi_{\ell_{2}, x}}{\left(\psi_{n}-\psi_{\ell_{1}}\right)\left(\psi_{n}-\psi_{\ell_{2}}\right)} \\
& +\left[\prod_{\ell=1, \ell \neq n}^{N}\left(\psi_{n}-\psi_{\ell}\right)\right]^{-1} \sum_{m=1}^{N}\left[a_{m} \varphi_{m, x} \varphi_{m}\left(\psi_{n}\right)^{N-m}\right],
\end{aligned}
$$

where the parameters $a_{m}$ are $N$ arbitrary (complex) numbers, the $N$ (complex) functions $\psi_{n} \equiv$ $\psi_{n}(x, t)$ are the dependent variables, and the $N$ (complex) functions $\varphi_{m} \equiv \varphi_{m}(x, t)$ respectively $\varphi_{m, x} \equiv \varphi_{m, x}(x, t)$ are expressed in terms of the dependent variables $\psi_{n} \equiv \psi_{n}(x, t)$ and their $x$ derivatives $\psi_{n, x} \equiv \psi_{n, x}(x, t)$ by the formulas (1.2) respectively (2.2).

This means that the initial-value problem-to compute the $N$ functions $\psi_{n}(x, t)$ for all time $t>0$ from given initial data $\psi_{n}(x, 0)$ — can be solved by algebraic operations (including changes of variables from the coefficients to the zeros of a polynomial of degree $N$ such as (1.1)) and via the standard Spectral Transform technique. The procedure to do so is detailed in the following Section 3 , and this implies the validity of the solutions reported below.

For $N=2$ this system of 2 coupled nonlinear PDEs reads as follows:

$$
\begin{aligned}
\psi_{n, t}+\psi_{n, x x x}= & \left(\psi_{n}-\psi_{n+1}\right)^{-1}\left\{3\left(\psi_{n, x x} \psi_{n+1, x}+\psi_{n, x} \psi_{n+1, x x}\right)\right. \\
& -\left[a_{1}\left(\psi_{n, x}+\psi_{n+1, x}\right)\left(\psi_{n}+\psi_{n+1}\right) \psi_{n}\right] \\
& \left.+a_{2}\left(\psi_{n, x} \psi_{n+1}+\psi_{n} \psi_{n+1, x}\right) \psi_{n} \psi_{n+1}\right\}, \quad n=1,2 \bmod (2) .
\end{aligned}
$$

An example of specific solution of this system of 2 coupled nonlinear PDEs, (2.6), reads as follows:

$$
\begin{aligned}
\psi_{n}(x, t)= & \left\{\frac{-6 \beta_{1}\left(\gamma_{1}\right)^{2}}{a_{1} \cosh ^{2}\left[\gamma_{1}\left(x-4 \gamma_{1} t\right)\right]}\right\} . \\
& \cdot\left\{1+(-1)^{n}\left[1-\frac{\left(a_{1}\right)^{2} \beta_{2}\left(\gamma_{2}\right)^{2} \cosh ^{4}\left[\gamma_{1}\left(x-4 \gamma_{1} t\right)\right]}{3 a_{2}\left(\beta_{1}\right)^{2}\left(\gamma_{1}\right)^{4} \cosh ^{2}\left[\gamma_{2}\left(x-4 \gamma_{2} t\right)\right]}\right]^{1 / 2}\right\}, \\
n=1,2, &
\end{aligned}
$$

where the 2 parameters $a_{n}$ are those appearing in the system (2.6) and the 4 (nonvanishing) parameters $\beta_{n}$ and $\gamma_{n}$ can be arbitrarily assigned. Note that if these 4 parameters are all real it is then sufficient that the parameter ratio $\beta_{2} / a_{2}$ be negative, $\beta_{2} / a_{2}<0$, for this solution to be real and nonsingular for all real values of the dependent variables $x$ and $t$. Also note that if $\gamma_{1}=\gamma_{2}=\gamma$ this solution has the "single soliton" feature to depend on the space and time coordinates only via their combination $x-4 \gamma t$.

For $N=3$ this system of 3 coupled nonlinear PDEs, (2.5), reads as follows:

$$
\begin{aligned}
\psi_{n, t}+\psi_{n, x x x}= & 3 \sum_{s=1,2}\left(\frac{\psi_{n, x x} \psi_{n+s, x}+\psi_{n, x} \psi_{n+s, x x}}{\psi_{n}-\psi_{n+s}}\right) \\
& +\left[\left(\psi_{n}-\psi_{n+1}\right)\left(\psi_{n}-\psi_{n+2}\right)\right]^{-1}\left\{-6\left[\psi_{n, x} \psi_{n+1, x} \psi_{n+2, x}\right]\right. \\
& \left.+\sum_{m=1}^{3}\left[a_{m} \varphi_{m, x} \varphi_{m}\left(\psi_{n}\right)^{N-m}\right]\right\}, n=1,2,3 \bmod (3),
\end{aligned}
$$


where of course $\varphi_{m}$ respectively $\varphi_{m, x}$ are given by (1.2) respectively (2.2) (with $N=3$ ).

\section{Proofs}

The proofs of the above two Propositions are actually quite easy. The starting point are the 3 identities [3]

$$
\begin{gathered}
\psi_{n, t}=-\left[\prod_{\ell=1, \ell \neq n}^{N}\left(\psi_{n}-\psi_{\ell}\right)\right]^{-1} \sum_{m=1}^{N}\left[\varphi_{m, t}\left(\psi_{n}\right)^{N-m}\right], \\
\psi_{n, x x}=\sum_{\ell=1, \ell \neq n}^{N}\left(\frac{2 \psi_{n, x} \psi_{\ell, x}}{\psi_{n}-\psi_{\ell}}\right)-\left[\prod_{\ell=1, \ell \neq n}^{N}\left(\psi_{n}-\psi_{\ell}\right)\right]^{-1} \sum_{m=1}^{N}\left[\varphi_{m, x x}\left(\psi_{n}\right)^{N-m}\right], \\
\psi_{n, x x x}=3 \sum_{\ell=1, \ell \neq n}^{N}\left(\frac{\psi_{n, x x} \psi_{\ell, x}+\psi_{n, x} \psi_{\ell, x x}}{\psi_{n}-\psi_{\ell}}\right) \\
-3 \sum_{\ell_{1}, \ell_{2}=1, \ell_{1} \neq \ell_{2}, \ell_{1}, \ell_{2} \neq n}^{N}\left[\frac{\psi_{n, x} \psi_{\ell_{1}, x} \psi_{\ell_{2}, x}}{\left(\psi_{n}-\psi_{\ell_{1}}\right)\left(\psi_{n}-\psi_{\ell_{2}}\right)}\right] \\
-\left[\prod_{\ell=1, \ell \neq n}^{N}\left(\psi_{n}-\psi_{\ell}\right)^{-1}\right] \sum_{m=1}^{N}\left[\varphi_{m, x x x}\left(\psi_{n}\right)^{N-m}\right],
\end{gathered}
$$

that relate the $N$ zeros $\psi_{n}$ and the $N$ coefficient $\varphi_{m}$ of a polynomial such as (1.1).

We now note that the sum of the first two of these identities imply the identity

$$
\begin{aligned}
\psi_{n, t}+\psi_{n, x x}= & \sum_{\ell=1, \ell \neq n}^{N}\left(\frac{2 \psi_{n, x} \psi_{\ell, x}}{\psi_{n}-\psi_{\ell}}\right) \\
& -\left[\prod_{\ell=1, \ell \neq n}^{N}\left(\psi_{n}-\psi_{\ell}\right)\right]^{-1} \sum_{m=1}^{N}\left\{\left[\varphi_{m, t}+\varphi_{m, x x}\right]\left(\psi_{n}\right)^{N-m}\right\}
\end{aligned}
$$

and likewise the sum of the first and third of the identities (3.1) implies the identity

$$
\begin{aligned}
\psi_{n, t}+\psi_{n, x x x}= & 3 \sum_{\ell=1, \ell \neq n}^{N}\left(\frac{\psi_{n, x x} \psi_{\ell, x}+\psi_{n, x} \psi_{\ell, x x}}{\psi_{n}-\psi_{\ell}}\right) \\
& -3 \sum_{\ell_{1}, \ell_{2}=1, \ell_{1} \neq \ell_{2}, \ell_{1}, \ell_{2} \neq n}^{N}\left[\frac{\psi_{n, x} \psi_{\ell_{1}, x} \psi_{\ell_{2}, x}}{\left(\psi_{n}-\psi_{\ell_{1}}\right)\left(\psi_{n}-\psi_{\ell_{2}}\right)}\right] \\
& -\left[\prod_{\ell=1, \ell \neq n}^{N}\left(\psi_{n}-\psi_{\ell}\right)^{-1}\right] \sum_{m=1}^{N}\left\{\left[\varphi_{m, t}+\varphi_{m, x x x}\right]\left(\psi_{n}\right)^{N-m}\right\} .
\end{aligned}
$$

Now assume that the $N$ functions $\varphi_{m} \equiv \varphi_{m}(x, t)$ satisfy the Burgers equations

$$
\varphi_{m, t}+\varphi_{m, x x}=a_{m} \varphi_{m, x} \varphi_{m}
$$

it is plain, see (3.2a), that this implies that the $N$ functions $\psi_{n} \equiv \psi_{n}(x, t)$ satisfy the system of PDEs (2.1). Proposition 2.1 is thereby proven. Indeed this implies that the solution of the initialvalue problem for this system of PDEs, (2.1), is yielded by the following procedure. Step (i): from 
the initial data $\psi_{n}(x, 0)$ compute the corresponding functions $\varphi_{m}(x, 0)$ (via the formulas (1.2)). Step (ii): solve the $C$-integrable PDEs (3.3) with these initial data $\varphi_{m}(x, 0)$, obtaining thereby the functions $\varphi_{m}(x, t)$ for all time $t>0$. Step (iii): the solutions $\psi_{n}(x, t)$ of the system of PDEs (2.1) are then provided by the $N$ zeros of the polynomial (1.1) with coefficients $\varphi_{m}(x, t)$. And of course the explicit solution (2.4) is manufactured using the single-soliton solutions of the Burgers equations (3.3).

The proof of Proposition 2.2, and the procedure to solve the system of PDEs (2.5), are quite analogous, except that the role of the identity (3.2a) is now played by the identity (3.2b), and the role played by the $C$-integrable Burgers PDEs (3.3) is now played by the $S$-integrable KdV PDEs

$$
\varphi_{m, t}+\varphi_{m, x x x}+a_{m} \varphi_{m, x} \varphi_{m}=0 .
$$

\section{Outlook}

It is plain that the approach employed in this paper provides the possibility to identify a large universe of new integrable/solvable systems of nonlinear PDEs; the two PDEs specifically discussed above are merely examples of the vistas opened by this methodology to identify new integrable/solvable systems of nonlinear PDEs. Note for instance that the assumptions made above- that all the coefficients $\varphi_{m}(x, t)$ satisfy the same integrable PDE-are not quite necessary; for instance in the case of Proposition 2.1 some of the coefficients $\varphi_{m}(x, t)$ might satisfy the C-integrable Kundu-Eckhaus PDE [6] and in the case of Proposition 2.2 some of the coefficients $\varphi_{m}(x, t)$ might satisfy the S-integrable Modified KdV PDE... Moreover, all the novel integrable/solvable PDEs identified via this approach can themselves be subsequently interpreted as characterizing the evolution of the coefficients of a polynomial, hence as inputs for the generation of new systems of integrable/solvable PDEs via this approach [4]; and it is also possible to extend this approach to a multidimensional context (beyond the $1+1$ context of the present paper) [2] and to more general auxiliary functions than polynomials [7].

This approach might moreover open the way to the identification and investigation of new integrable/solvable systems of nonlinear PDEs which are of interest because of their universality hence possible wide applicability (for these notions see for instance [8] and references therein).

\section{References}

[1] F. Calogero, "Motion of Poles and Zeros of Special Solutions of Nonlinear and Linear Partial Differential Equations, and Related "Solvable" Many Body Problems", Nuovo Cimento 43B, 177-241 (1978); Classical many-body problems amenable to exact treatments, Lecture Notes in Physics Monograph m66, Springer, 2001 (749 pages); Isochronous systems, Oxford University Press, 2008 (264 pages) (an updated paperback edition has been published by OUP in September 2012).

[2] F. Calogero, "Integrable nonlinear evolution equations and dynamical systems in multidimensions", in: $K d V$ '95, Proceedings of the International Symposium held in Amsterdam, The Netherlands, April 2326,1995 (edited by M. Hazewinkel, H.W. Capel and E.M. de Jager), also in Acta Applicandae Mathematicae 39, 229-244 (1995). F. Calogero and M. Sommacal, "Solvable nonlinear evolution PDEs in multidimensional space", SIGMA 2, 088 (2006) (17 pages), nlin.SI/0612019.

[3] F. Calogero, "New solvable variants of the goldfish many-body problem", Studies Appl. Math. 137 (1), 123-139 (2016), DOI: 10.1111/sapm.12096. O. Bihun and F. Calogero, "Novel solvable many-body problems", J. Nonlinear Math. Phys. 23, 190-212 (2016), DOI: 10.1080/14029251.2016.1161260. M. Bruschi and F. Calogero, "A convenient expression of the time-derivative $z_{n}^{(k)}(t)$, of arbitrary order $k$, of the zero $z_{n}(t)$ of a time-dependent polynomial $p_{N}(z ; t)$ of arbitrary degree $N$ in $z$, and solvable dynamical systems", J. Nonlinear Math. Phys. 23, 474-485 (2016). 
[4] O. Bihun and F. Calogero, "A new solvable many-body problem of goldfish type", J. Nonlinear Math. Phys. 23, 28-46 (2016), arXiv:13749 [math-ph], DOI:10.1080/14029251.2016.1135638; "Generations of monic polynomials such that the coefficients of each polynomial of the next generation coincide with the zeros of a polynomial of the current generation, and new solvable many-body problems", Lett. Math. Phys. 106 (7), 1011-1031 (2016), DOI: 10.1007/s11005-016-0836-8. arXiv: 1510.05017 [math-ph]; "Generations of solvable discrete-time dynamical systems", J. Math. Phys. (submitted to, 2016.06.23). F. Calogero, "A solvable $N$-body problem of goldfish type featuring $N^{2}$ arbitrary coupling constants", J. Nonlinear Math. Phys. 23, 300-305 (2016), DOI: 10.1080/14029251.2016.1175823; "Novel isochronous $N$-body problems featuring $N$ arbitrary rational coupling constants", J. Math. Phys. 57, 072901 (2016), http://dx.doi.org/10.1063/1.4954851; "Three new classes of solvable $N$-body problems of goldfish type with many arbitrary coupling constants", Symmetry 8, 53 (2016), DOI:10.3390/sym8070053; "Yet another class of new solvable $N$-body problems of goldfish type", Qualit. Theory Dyn. Syst. (in press); "New solvable dynamical systems", J. Nonlinear Math. Phys. 23, 486-493 (2016).

[5] C. S. Gardner, J. M. Greene, M. D. Kruskal, R. M. Miura, "'Method for Solving the Korteweg-deVries Equation", Phys. Rev. Lett. 19, 1095-1097 (1967); DOI: 10.1103/PhysRevLett.19.1095.

[6] A. Kundu, "Landau-Lifshitz and higher-order nonlinear systems gauge generated from nonlinear Schrödinger-type equations", J. Math. Phys. 25, 3433-3438 (1984). F. Calogero and S. De Lillo, "The Eckhaus PDE i $y_{t}+y_{x x}+2\left(|y|^{2}\right)_{x} y+|y|^{4} y=0$ ", Inverse Problems 3, 633-681 (1987); Erratum 4, 571 (1988).

[7] F. Calogero, J.-P. Françoise and M. Sommacal, "Solvable nonlinear evolution PDEs in multidimensional space involving trigonometric functions", J. Phys. A: Math. Theor. 40, F363-F368 (2007); "Solvable nonlinear evolution PDEs in multidimensional space involving elliptic functions", J. Phys. A: Math. Theor. 40, F705-F711 (2007).

[8] F. Calogero, "Universal C-Integrable Nonlinear Partial Differential Equation in $N+1$ Dimensions", J. Math. Phys. 34, 3197-3209 (1993). 